\title{
Prognostic significance of perigastric tumor deposits in patients with primary gastric cancer
}

\author{
Shrestha Anup ${ }^{1,2+}$, Jun Lu ${ }^{1,2+}$, Chao-Hui Zheng ${ }^{1,2}$, Ping Li ${ }^{1,2}$, Jian-Wei Xie ${ }^{1,2}$, Jia-Bin Wang ${ }^{1,2}$, Jian-Xian Lin ${ }^{1,2}$, \\ Qi-Yue Chen ${ }^{1,2}$, Long-Long Cao ${ }^{1,2}$, Mi Lin ${ }^{1,2}$, Qian Yu ${ }^{3}$, Ying-Hong Yang ${ }^{3}$ and Chang-Ming Huang ${ }^{1,2^{*}}$
}

\begin{abstract}
Background: The presence and the prognostic significance of perigastric tumor deposits (TDs) in primary gastric cancer have not been extensively studied. The aim of this study was to evaluate the prognostic significance perigastric TDs in primary gastric cancer.

Methods: From 2005 to 2010, 1250 patients underwent R0 gastrectomy at the Department of Gastric Surgery, Fujian Medical University Union Hospital, Fuzhou, China. Out of 1250 patients, 132 patients with perigastric TDs were identified. Additionally, 132 patients with staged matched gastric cancer without tumor deposits were selected as a control group.

Results: Perigastric TDs were observed in 132 (10.5\%) of the 1250 patients with gastric cancer who underwent R0 gastrectomy. There were 94 males (71.21\%) and 38 females (28.79\%) (2.47:1). The mean age was 57.21 years. Clinicopathologic characteristics between the two groups matched well. There was a significant difference in the overall survival of those with and without TDs by univariate $(p<0.05)$ and multivariate $(p<0.05)$ survival analysis. The 1-, 3-and 5-year overall survival rates of patients with TDswere69.6\%, 39.3\%, and 24.2\%, respectively, and were significantly poorer than those of the staged matched control group. There was no correlation between the number of TDs and patient survival in patients with gastric cancer ( $p>0.05)$; however, when comparing each $p T$ tumor group with the perigastric TD group, the stage T4 survival rate was very similar to that observed in patients with TDs.

Conclusions: Perigastric TDs are an independent predictive prognostic factor for gastric cancer and may be appropriately considered a form of serosal invasion. We suggest that TDs should be included in TNM staging system for better outcomes.
\end{abstract}

Keywords: Gastric cancer, Perigastric tumor deposits, Survival, Prognostic significance

\section{Background}

Gastric cancer is an aggressive and mostly lately diagnosed disease that continues to have significant impact on cancer related death worldwide. Because most of the patients presents with advanced disease, it has a high mortality rate, making it the second leading cause of

\footnotetext{
* Correspondence: hcmlr2002@163.com

${ }^{\dagger}$ Equal contributors

'Department of Gastric Surgery, Fujian Medical University Union Hospital, Fuzhou, China

${ }^{2}$ Department of General Surgery, Fujian Medical University Union Hospital,

Fuzhou, China

Full list of author information is available at the end of the article
}

cancer-related death worldwide (http://globocan.iarc.fr/ Pages/fact_sheets_cancer.aspx, [1]). It is especially prevalent in East Asia and South America and has been increasing in developing countries.

The accurate staging of gastric cancer is very important for treatment plan and prognosis. The TNM(Tumor, Lymph Nodes and Metastasis) staging system for gastric cancer is based on the infiltration depth of the primary tumor $(\mathrm{pT})$, the number of metastatic lymph nodes $(\mathrm{pN})$ and the presence of distant metastasis (pM) [2]. In the recent years, several other prognostic factors, such as histological type and lymphovascular invasion, intramural 
carcinomatosis of the lymph vessels etc., have been identified as significant and even as independent predictors for survival [3].

Tumor deposits (TDs), first recognized by Gabriel in 1935 [4], are defined as cluster of peritumoral nodules in the peritumoral adipose tissue of a primary carcinoma without histologic evidence of residual lymph node in the nodule. These may represent discontinuous spread, venous invasion with the extravascular spread, or a totally replaced lymph node [2]. The prognostic significance of tumor deposits in the colon and rectal cancers have been confirmed by several studies [2, 5-9]. Puppa et al. reported that TDs were not limited to colorectal cancers, but were also common in other tumor types including biliary duct, ovarian, gastric and pancreatic cancers [10].

The recently released 7th edition of the American Joint Committee on Cancer (AJCC) staging system for gastric cancer does not distinguish between lymph node metastasis and perigastric TDs. The AJCC staging for gastric cancer considers all perigastric metastatic nodules without evidence of residual lymph node tissue to be regional lymph node metastases [5]. The presence and the prognostic significance of TDs in gastric cancer have not been extensively studied or well documented. To date, there are only 3 series about gastric cancer tumor deposits (TDs) with prognostic implications [11-13]. The objective of this study was to investigate the prognostic significance of TDs in gastric cancer patients who underwent R0 gastrectomy and to determine whether they should be included in the TNM staging system of gastric cancer.

\section{Methods}

This study utilized a prospectively maintained database of patients who underwent R0 gastrectomy at the Department of Gastric surgery, Fujian Medical University Union Hospital, Fuzhou, China, from January 2005 to December 2010. Of patients who underwent R0 gastrectomy for primary gastric cancer, the patients with perigastric tumor deposits were included in this study. A tumor deposit was defined as a discrete focus of tumor found in the perigastric fat or in an adjacent ligament away from the leading edge of the tumor and showing no evidence of residual lymph node tissue but within the drainage area of the primary cancer $[11,12]$. An identical number of patients without TDs who underwent R0 gastrectomy during the same period and matched with same TNM stage were included as a reference group for prognostic comparison to those with TDs. No patient received neoadjuvant therapy or radiation pre-operatively. According to the Japanese Gastric Cancer Treatment Guidelines, adjuvant chemotherapy with 5-fluorouracil (5-FU)-based regimens (mostly 5-FU with cisplatin) has been administered to all patients with stage II/III GC at our institution, unless contraindicated by a patient's condition or their refusal.

The tumor was classified according to the 7th edition AJCC staging system for gastric cancer. Clinicopathologic features such as gender, age at the time of diagnosis, differentiation type, size, location, AJCC pT category, the number of node metastases, AJCC $\mathrm{pN}$ category, and the type of operation were compared between the patients with and without TDs. Overall survival rates were compared between the patients with and without TDs and among patients with a different number of TDs. For each patient within the $\mathrm{pT}$ category, the prognosis of those with and without TDs was compared.

\section{Follow-up}

Routine follow-up consisted of physical examination, laboratory tests, chest X-ray, abdominal and pelvic ultrasonography and computed tomography scan. Patients were followed-up every 3 months during the first year and every 6 or 12 months thereafter, for a total of 5 years. Endoscopy was performed every 1 year. All surviving patients were followed for more than 5 years. Overall survival was calculated from the date of diagnosis to last contact, date of death, or date when the survival information was collected.

\section{Statistical analysis}

All statistical analyses and graphics were performed with the IBM SPSS version 23.0 statistical package (International Business Machines Corp., New Orchard Road Armonk, New York 10,504 914-499-1900, USA) for Windows. For comparisons of clinicopathologic characteristics between the two-propensity score-matched groups, the Chi-squared test or Fisher's exact test were used for categorical variables as appropriate, and Student's t-test was used for quantitative variables. Overall survival rates were determined using the Kaplan-Meier estimator, with an event being defined as death from cancer-related causes. The log-rank test was used to identify differences between the survival curves of different patient groups. In the univariate analysis, the 2-tailed Chi-square or 2-tailed t-test was used for statistical comparisons. In the multivariate analysis, Cox's proportional hazard model was used to identify independent factors correlated with prognosis. The confidence interval (CI) method was used to compare differences in means between the predictive accuracy estimates for models that either included or did not include TDs. All $p$ values were two-sided with $p$ values $<0.05$ considered statistically significant.

\section{Results}

Patients' demographics

TDs were observed in 132 (10.5\%) out of 1250 patients with gastric cancer who underwent R0 gastrectomy from 
January 2005 to December 2010.There were 94 male and 38 female patients with TDs (mean age of 57.21 years) and 100 male and 32 female patients without TDs (mean age of 58.64 years). Clinicopathologic characteristics of the gastric cancer patients with TDs were compared with those of the patients without TDs (Additional file 1: Table S1).

\section{Survival analysis}

1. There was a significant difference between the Kaplan-Meier curves for those patients with and without TDs $(p=0.008)$ (Fig. 1). The 1-year, 3-year, and 5-year survival rates of patients with tumor deposits were $69.6 \%, 39.3 \%$, and $24.2 \%$, respectively, and those for patients without TDs were $91.7 \%$, $46.6 \%$, and $38.5 \%$, respectively. We also performed a subgroup analysis regarding $\mathrm{pT}$ and $\mathrm{pN}$ stage (Fig. 2, and Additional file 2: Table S2). There was no correlation between the number of TDs and patient survival in patients with gastric cancer $(p>0.05)$

(Additional file 3: Fig. S1).

2. Univariate analysis:

Of the 8 clinicopathological variables identified by univariate analysis, the statistically significant prognostic factors for survival in all patients were tumor size $(P=0.014)$, depth of invasion $(P \leq 0.001)$, lymph node metastasis $(p \leq 0.001)$, histologic grading $(P=0.014)$, and TDs $(P=0.010)$ (Table 1$)$.

\section{Multivariate analysis:}

To adjust for possible confounding baseline factors, Cox multivariate analysis was performed. Multivariate analysis of the five prognostic factors, using Cox's proportional hazard regression model, showed that depth of invasion, lymph node metastasis, and perigastric TDs were each independent statistically significant prognostic factors $(p<0.05$ each) (Table 1$)$.

4. To further elucidate the prognostic impact of TDs on gastric cancer patients who underwent R0 gastrectomy, the Kaplan-Meier survival curves were compared between patients with TDs and each $\mathrm{pT}$ category without TDs. The differences in the Kaplan-Meier survival curve were observed between different $\mathrm{pT}$ categories without TDs and with TDs. Regardless of the $\mathrm{T}$ stage, the patients with TDs has the worst prognosis; however, the survival rate of patients with TDs and stage T4 disease without tumor deposit was similar (Fig. 3, and Additional file 2: Table S2).

\section{Discussion}

Even though there has been a vast improvement in the overall survival rate of patients with gastric cancer in past few decades, many questions are yet to be answered regarding the histopathological and predictive factors. Studies have demonstrated that the presence of TDs has independent prognostic value in colorectal cancer;

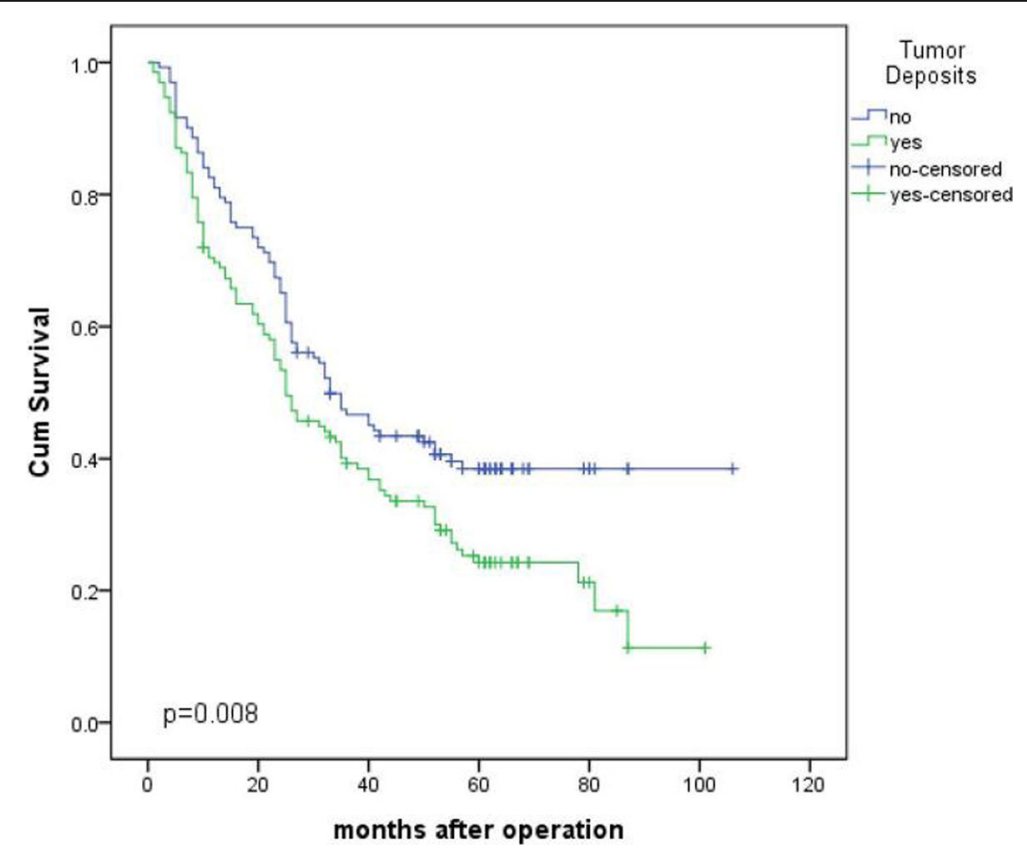

Fig. 1 Comparison of survival curves between patients with tumor deposits (TDs) and without TDs 


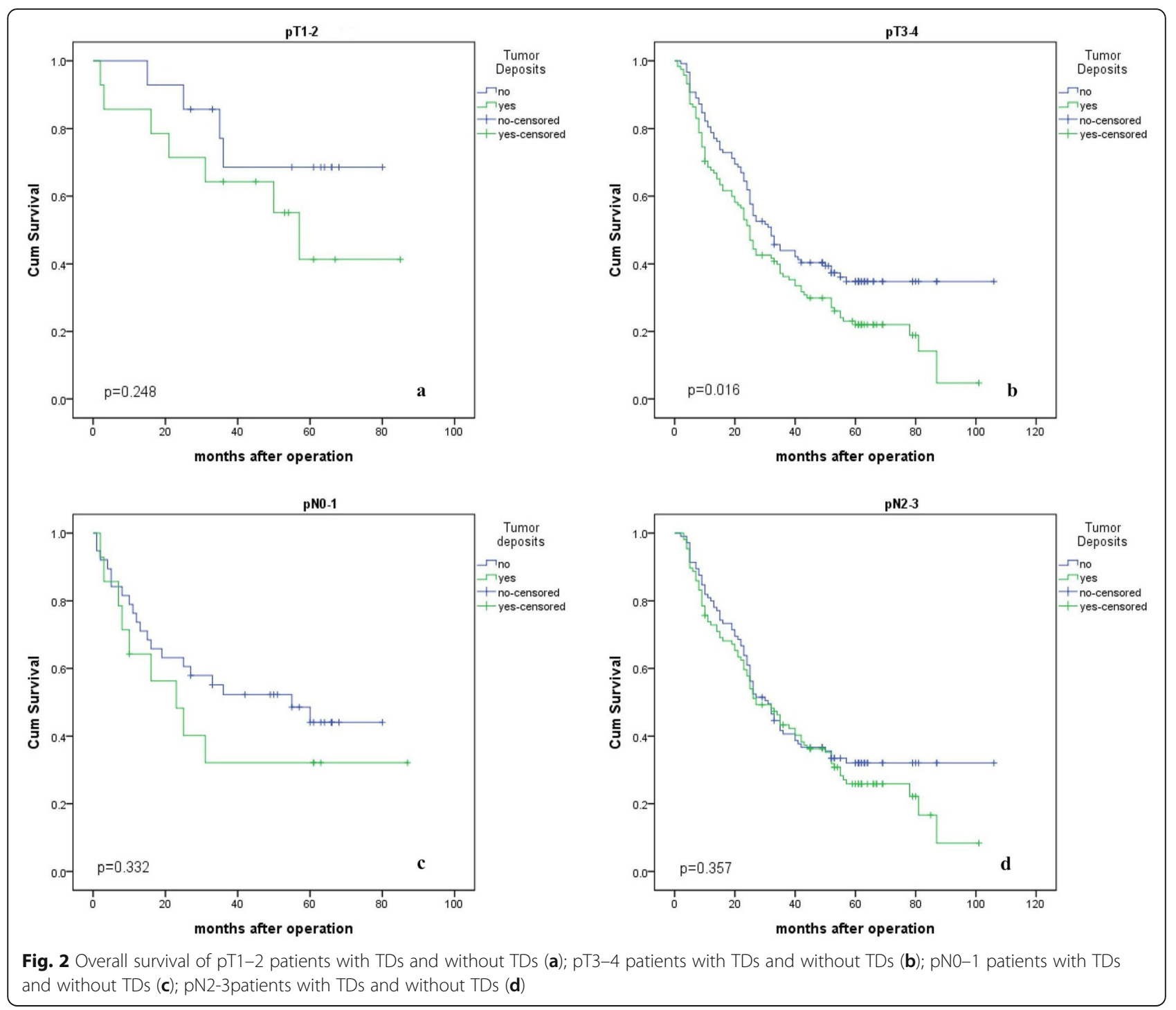

however, only a few studies have focused on this issue in gastric cancer.

TDs are currently defined as focal clusters of tumor cells located in the serosal fat, discontinuous with the primary tumor and unassociated with a lymph node [14]. Although they are encountered in some types of adenocarcinoma, including gastric, biliary, and pancreatic $[12,13]$ the prognostic significance of TDs has been studied mainly in colorectal carcinomas [8, 14-20]. Currently, TDs are part of the TNM classification of colorectal carcinomas [5].

In this study, we investigate the prognostic significance of TDs in primary gastric cancer. Only 3 studies to date have been identified that focus on the prognostic value of TDs in gastric cancer. TDs were found in $17.8 \%$, $23.9 \%$, and $24 \%$ of cases, in the order of the date published. Sun et al. reported that TDs were more frequently observed in cancers of larger size, Borrmann type 4 , in cases with lymphovascular invasion, with deeper depth of invasion and with extended lymph node metastasis, and TDs were significantly correlated to gastric cancer patients' survival. Because of this, it might be more suitable for TDs to be treated as a form of serosal invasion. Consequently, en bloc resection of the primary carcinoma is important, and adjuvant chemotherapy should always be considered if TDs have been detected. A revised pT category, in which all cancers with TDs in pT1-4a category are incorporated into those without TDs in pT4a category, was also recommended [12].

In contrast to Sun et al., Lee et al. stated that perigastric TDs are a strong prognostic predictor in gastric cancer, and recommended TDs to be included in lymph node staging. In addition, they adopted the definition of lymph node metastasis from the AJCC (7th ed.) staging 
Table 1 Univariate and multivariate survival analysis of the patients following operation for gastric cancer

\begin{tabular}{|c|c|c|c|c|c|c|}
\hline \multirow[t]{2}{*}{ Variable } & \multicolumn{3}{|c|}{ Univariate analysis } & \multicolumn{3}{|c|}{ Multivariate analysis } \\
\hline & $\overline{\mathrm{HR}}$ & $95 \% \mathrm{Cl}$ & $P$ & $\overline{\mathrm{OR}}$ & $95 \% \mathrm{Cl}$ & $P$ \\
\hline Gender(M vs F) & 1.250 & $0.889-1.758$ & 0.199 & / & / & $/$ \\
\hline Age (<60 yrs. vs $\geq 60$ yrs) & 0.942 & $0.702-1.265$ & 0.692 & l & l & l \\
\hline Location & & & 0.351 & / & / & l \\
\hline Middle vs Upper & 0.695 & $0.402-1.204$ & & & & \\
\hline Lower vs Upper & 0.958 & $0.616-1.489$ & & & & \\
\hline Whole vs Upper & 1.107 & $0.766-1.601$ & & & & \\
\hline Tumor size $(<5 \mathrm{~cm}$ vs $\geq 5 \mathrm{~cm})$ & 1.559 & $0.096-2.217$ & 0.014 & 1.198 & $0.820-1.753$ & 0.351 \\
\hline Histologic type (Differentiated vs Un-) & 0.657 & $0.470-0.920$ & 0.014 & 0.766 & $0.541-1.084$ & 0.133 \\
\hline Lymph Node Metastasis & & & $<0.001$ & & & 0.009 \\
\hline N1 vs N0 & 0.497 & $0.292-0.847$ & & 0.770 & $0.432-1.372$ & \\
\hline N2 vs N0 & 0.817 & $0.465-1.436$ & & 0.889 & $0.502-1.577$ & \\
\hline N3 vs NO & 0.504 & $0.361-0.704$ & & 0.554 & $0.393-0.780$ & \\
\hline Depth of Invasion & & & $<0.001$ & & & 0.001 \\
\hline T2 vs T1 & 0.114 & $0.061-0.816$ & & 0.112 & $0.015-0.816$ & \\
\hline T3 vs T1 & 0.817 & $0.226-0.819$ & & 0.480 & $0.241-0.954$ & \\
\hline T4 vs T1 & 0.504 & $0.393-0.745$ & & 0.570 & $0.396-0.818$ & \\
\hline Tumor Deposits (present vs absent) & 1.475 & $1.098-1.981$ & 0.010 & 1.411 & $1.029-1.936$ & 0.033 \\
\hline
\end{tabular}

system for colorectal carcinoma and restaged gastric cancer cases after excluding TDs from being counted as lymph node metastases. In patients with the same $\mathrm{pN}$ stage, the presence of TDs was significantly associated with a poorer prognosis. In that study, there were two pT1 cases with TDs and four pT2 with TDs, which were only $0.9 \%$ (6 of a total of 653 patients). Therefore, $\mathrm{TD}$ is suggested to be less important in clinical pT staging [11].

In the most recent study, Ersen et al. reported that TDs were strongly correlated to histologic type of the primary tumor and the presence of vascular invasion.

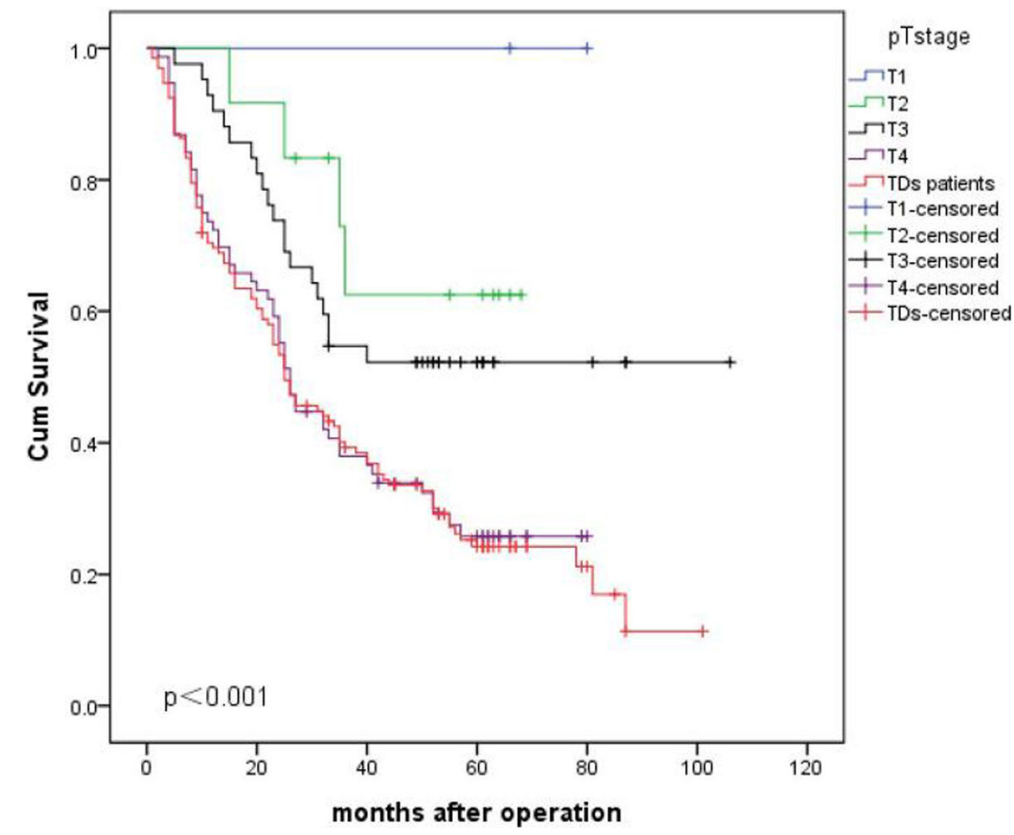

Fig. 3 Comparison of survival curves between patients with TDs and those without TDs in PT1-4a category 
TDs were more common in intestinal type carcinomas. Compared to other studies, there was no statement about the placement of TDs as an independent prognostic factor in TMN staging [13].

In our current study, TDs were observed in 132 (10.5\%) of 1250 patients with gastric cancer who underwent R0 gastrectomy. The number of TDs were as follows: 99 patients with $1 \mathrm{TD}, 23$ patients with 2 TDs, and 11 patients with more than 3 TDs. Sun et al. concluded that increasing numbers of TDs are associated with a poor prognosis [12]. In contrast to Sun et al., our results did not show any significant difference in the prognosis of patients with different numbers of TDs. There was no significant difference in the Kaplan Meier survival curve between different numbers of TDs. Belt et al. described that there was no correlation between the number of TDs and the recurrence rate in stage II patients in colorectal cancers [8], and we describe the same for the prognosis in patients with gastric cancers. It has been suggested that the lymphatic invasion is significantly associated with a poorer overall survival in node-negative gastric cancer patients [21]. But our study also suggests that the correlation between the lymphatic invasion and presence of tumor deposits was not significance. Even though our results show there are less significant role of lymphatic invasion in tumor deposits, we do believe it have significant role. Therefore, extensive detailed study should be done in the future regarding this matter.

To illustrate the effect of TDs in the prognosis of patients with gastric cancer we compared the 5-, 3-and 1 -year survival rates between patients with and without TDs. We then used the Kaplan Meier curves to test the prognostic significance of perigastric TDs in gastric cancer. Our results show that the patients with perigastric TDs had a poorer prognosis than those without TDs. Multivariate Cox Regression analysis also confirmed the prognostic significance of TDs. Its hazard ratio was higher than other clinicopathologic predictive factors. This proves that TDs can be used as an independent factor for survival prognosis.

The inclusion of TDs in the TNM staging system in gastric cancer is still a matter of further study. Whether to incorporate TDs in the $\mathrm{pN}$ or $\mathrm{pT}$ category if these are to be considered in TNM staging is a matter of debate. The TDs in the colon and rectum have been well placed in the 7th edition of TNN system. The tumors with TDs are classified as the pN1c category if no lymph node metastasis has been detected. Puppa G. et al., suggested that TDs in gastric cancer are peritoneal seeding from either primary tumors or metastatic lymph nodes [10]. In this study, we placed all patients with perigastric TDs in one group regardless of their $\mathrm{pT}$ category, and we configured the Kaplan-Meier survival curves for the TD group and each pT category. A significant difference in overall survival was observed in between the T1, T2, T3 and TDs groups, but there was a similarity between the T4 and TDs groups (Fig. 3), suggesting that the prognosis of patients with perigastric TDs was as poor as patients with advanced T4 stage, regardless of the pT staging. Because of this, we suggest that perigastric TDs are included in pT staging, and patients with TDs should be treated separately from those without TDs when staging. Our results indicate that it might be more suitable for TDs to be considered as a form of serosal invasion; therefore, TDs should be treated as pT4a stage disease. The placement of TDs in the pT category is very effective and accurate in implications for patient prognosis and staging. These results also indicate that gastric cancer with TDs shows aggressive behavior and adjuvant chemotherapy should always be considered after en bloc resection of the tumor to achieve the better outcomes even for pT1 patients. Our results show that pT1 patients without TDs had good prognosis, but patients with TDs had worse prognosis, so even early stage gastric cancer with TDs should be considered as a highly-advanced disease and should be treated differently than those without TDs. According to our results, patients in the pT1 category with TDs had similar survival rate as patients in the pT4 category without TDs; therefore, patients with TDs should be treated as patients with advanced stage disease.

There were some limitations during the study. First, this is a retrospective study. Lauren classification was not available before 2007 in our database. Therefore, we can't analyze the Lauren classification in the present study. Second, the numbers of pT1, pT2, and pT4b patients were low. Last but not the least, this single study from the east should also be verified in western patients. Further studies still need to be conducted.

\section{Conclusion}

We drew the conclusion that the presence of TDs is a strong prognostic factor in gastric cancer and should be considered an independent predictive factor for prognosis. It should be considered in TNM classification as a pT4 disease.

\section{Additional files}

Additional file 1: Table S1. Clinicopathologic characteristics of gastric cancer patients with and without TDs. (DOC $69 \mathrm{~kb}$ )

Additional file 2: Table S2. Comparison of $\mathrm{T}$ - and $\mathrm{N}$ - staging with different tumor deposits (TDs) factors in terms of their power to stratify patients according to overall survival (OS). (DOCX $17 \mathrm{~kb}$ )

Additional file 3: Figure S1. Comparison of survival curves among patients with different number of tumor deposits. (JPEG 26 kb) 


\section{Abbreviations}

AJCC: American Joint Committee for Cancer; pM: distant metastasis; pN: the number of metastatic lymph nodes; $\mathrm{PT}$ : depth of the primary tumor;

TD: Tumor Deposit; TNM: Tumor, Lymph Nodes and Metastasis

\section{Acknowledgements}

The authors thank all the medical staff who contributed to the maintenance of the medical record database.

\section{Funding}

This work was supported by the National Key Clinical Specialty Discipline Construction Program of China (No. [2012] 649); Key Projects of Science and Technology Plan of Fujian Province (No. 2014Y0025); and Youth scientific research subject of Fujian provincial health and family planning commission (No. 2015-1-37). The funding body had no role in the study design, data collection and analysis, or preparation of manuscript.

\section{Availability of data and materials}

The data that support the findings of this study are available from the corresponding author upon reasonable request.

\section{Authors' contributions}

$J \mathrm{~L}$ and SA carried out the main manuscript writing and design. $\mathrm{CMH}$ and $\mathrm{CHZ}$ performed the operations. JL and SA collected and analyzed the data. $J L$ and SA wrote the manuscript and PL, JWX, JBW, JXL, QYC, LLC, and ML assisted in drafting the manuscript and reviewed the article. QY and YHY reviewed all the pathological specimens. All authors read and approved the final manuscript.

\section{Ethics approval and consent to participate}

Written consent was given by patients for the information to be stored in the hospital database and used for the research. This study was approved by our institutional review board, Fujian Medical University Union Hospital, Fuzhou, China.

\section{Consent for publication}

Written consent was given by the patients and their relatives to use their information in a research study and publish it.

\section{Competing interests}

The authors declare that they have no competing interests.

\section{Publisher's Note}

Springer Nature remains neutral with regard to jurisdictional claims in published maps and institutional affiliations.

\section{Author details}

'Department of Gastric Surgery, Fujian Medical University Union Hospital, Fuzhou, China. ${ }^{2}$ Department of General Surgery, Fujian Medical University Union Hospital, Fuzhou, China. ${ }^{3}$ Department of Pathology, Fujian Medical University Union Hospital, Fuzhou, China.

\section{Received: 23 March 2017 Accepted: 7 July 2017}

\section{Published online: 19 July 2017}

\section{References}

1. Global Cancer Facts \& Figures, 3rd ed. American Cancer Society. Available at http://www.cancer.org/downloads/STT/Global_Facts_and_Figures_2007_ rev2.pdf. Accessed 21 May 2015.

2. Edge $S B$, Fritz AG, Byrd DR, et al. AJCC cancer staging manual. 7th ed. New York: Springer; 2010.

3. Gurzu S, Orlowska J, Sugimura H, et al. Immunohistochemical features and staging of early gastric cancer. Archives of Medical Science. 2016;12:1-10.

4. Gabriel WB, Dukes C, Bussey HJR. Lymphatic spread in cancer of the rectum. Br J Surg. 1935;23:395-413.

5. Ono C, Yoshinaga K, Enomoto $M$, et al. Discontinuous rectal cancer spread in the mesorectum and the optimal distal clearance margin in situ. Dis Colon Rectum. 2002;45:744-9

6. Tateishi S, Arima S, Futami K, et al. A clinicopathological investigation of "tumor nodules" in colorectal cancer. Surg Today. 2005;35:377-84.
7. Goldstein NS, Turner JR. Pericolonic tumor deposits in patients with T3N+M0 colon adenocarcinomas. Cancer. 2000;88:2228-38.

8. Belt EJ, van Stijn MF, Bril H, et al. Lymph node negative colorectal cancers with isolated tumor deposits should be classified and treated as stage III. Ann Surg Oncol. 2010;17:3203-11.

9. Tong LL, Gao P, Wang ZN, et al. Is the seventh edition ofthe UICC/AJCC TNM staging system reasonable for patients with tumor deposits in colorectal cancer? Ann Surg. 2012;255:208-13.

10. Puppa G, Ueno H, Kayahara M, Capelli P, Canzonieri V, Colombari R, Maisonneuve P, Pelosi G. Tumor deposits are encountered in advanced colorectal cancer and other adenocarcinomas: an expanded classification with implications for colorectal cancer staging system including a unifying concept of in-transit metastases. Mod Pathol. 2009:22:410-5.

11. Lee HS, Lee HE, Yang Y-K, Kim WH. Perigastric tumor deposits in primary gastric cancer: implication for patient's prognosis and staging. Ann Surg Oncol. 2013;20:1604-13.

12. Sun Z, Wang ZN, Xu YY, Zhu GL, Huang BJ, Xu Y, Liu FN, Zhu Z, Xu HM. Prognostic significance of tumor deposits in gastric cancer patients who underwent radical surgery. Surgery. 2012;151:871-81.

13. Ersen A, Unlu MS, Akman T, Sagol O, Oztop I, Atila K, Bora S, Ellidokuz H, Sarioglu S. Tumor deposits in gastric carcinomas. Pathol Res Pract. 2014:9:565-70.

14. Nagtegaal ID, Quirke P. Colorectal tumor deposits in the mesorectum and pericolon: a critical review. Histopathology. 2007;51:141-9.

15. Nagtegaal ID, Tot $T$, Jayne $D G$, McShane $P$, Nihlberg A, Marshall $H C$, Påhlman L, Brown JM, Guillou PJ, Quirke P. Lymph nodes, tumor deposits, and TNM: are we getting better? J Clin Oncol. 2011;29:2487-92.

16. Ratto C, Ricci R, Rossi C, Morelli U, Vecchio FM, Doglietto GB. Mesorectal microfoci adversely affect the prognosis of patients with rectal cancer. Dis. Colon Rectum. 2002;45:733-42.

17. Shimada Y, Takii Y. Clinical impact of mesorectal extranodal cancer tissue in rectal cancer: detailed pathological assessment using whole-mount sections. Dis Colon Rectum. 2010:53:771-8.

18. Tong $L L$, Gao $P$, Wang $Z N$, Song $Y X, X u Y Y$, Sun $Z$, Xing $C Z$, Xu HM. Is the seventh edition of the UICC/AJCC TNM staging system reasonable for patients with tumor deposits in colorectal cancer? Ann Surg. 2012;255:208-13.

19. Ueno H, Mochizuki H. Clinical significance of extra bowel skipped cancer infiltration in rectal cancer. Surg Today. 1997;27:617-22.

20. Ueno $H$, Mochizuki $H$, Hashiguchi $Y$, Ishiguro M, Miyoshi M, Kajiwara $Y$, Sato T, Shimazaki H, Hase K. Extramural cancer deposits without nodal structure in colorectal cancer: optimal categorization for prognostic staging. Am J Clin Pathol. 2007;127:287-94.

21. Ichikawa D, Kubota T, Kikuchi S, Fujiwara H, Konishi H, Tsujiura M, Ikoma H, Nakanishi M, Okamoto K, Sakakura C, Ochiai T, Kokuba Y, Otsuji E. Prognostic impact of lymphatic invasion in patients with node-negative gastric cancer. J Surg Oncol. 2009;100:111-4.

\section{Submit your next manuscript to BioMed Central and we will help you at every step:}

- We accept pre-submission inquiries

- Our selector tool helps you to find the most relevant journal

- We provide round the clock customer support

- Convenient online submission

- Thorough peer review

- Inclusion in PubMed and all major indexing services

- Maximum visibility for your research

Submit your manuscript at www.biomedcentral.com/submit

\section{) BioMed Central}

